\title{
Updating the International Index of Erectile Function: evaluation of a large clinical data set
}

\author{
Andrew J. Vickers, PhD, \\ Department of Epidemiology and Biostatistics, Memorial Sloan Kettering Cancer Center, New \\ York, NY
}

\author{
Amy L. Tin, MA, \\ Department of Epidemiology and Biostatistics, Memorial Sloan Kettering Cancer Center, New \\ York, NY
}

Karandeep Singh, MD, University of Michigan Department of Urology, Ann Arbor, MI

Rodney L. Dunn, MS, University of Michigan Department of Urology, Ann Arbor, MI

John Mulhall, MD

Department of Surgery Memorial Sloan Kettering Cancer Center, New York, NY

\begin{abstract}
Introduction: The International Index of Erectile Function (IIEF) is the predominant patientreported outcomes instrument for assessing male sexual function. There are obvious problems with the use of the IIEF in the assessment of an individual patient, such as for men who use injections and men who do not engage in intercourse. The aim of the current study is Aim: To redesign the erectile function domain of the IIEF (IIEF6) to more accurately assess the individual patient.
\end{abstract}

Methods: In an observational study of men undergoing treatment for prostate cancer at a tertiary care institution, including 24,732 questionnaires completed by 6,780 individuals, IIEF6 scores were compared for patients using and not using erectile aids. Men not engaging in sexual intercourse were asked to describe the reason.

Main outcome measures: IIEF6 scores.

Results: Mean scores before erectile aids items were added was 17.7 compared to predicted scores of 18.3 vs. 16.7 if patients reported their function with vs. without the use of aids. No intercourse was reported for $35 \%$ of surveys. Reasons given were lack of ability or confidence in

Corresponding author: Andrew Vickers, Memorial Sloan Kettering Cancer Center, 485 Lexington Avenue, New York, NY, 10017, USA, vickersa@mskcc.org, Phone: +1-646-888-8233.

Conflict of interest: None

Publisher's Disclaimer: This is a PDF file of an unedited manuscript that has been accepted for publication. As a service to our customers we are providing this early version of the manuscript. The manuscript will undergo copyediting, typesetting, and review of the resulting proof before it is published in its final form. Please note that during the production process errors may be discovered which could affect the content, and all legal disclaimers that apply to the journal pertain. 
$53 \%$, lack of willing and available partner in $28 \%$, "other" in $17 \%$ (including respondent's or partner's health issues, low libido, preference for non-penetrative sex or for sex with men). Doubling the sum of the 3 non-intercourse IIEF6 questions had excellent properties (difference of 0.06 , limits of agreement -3.10 to 3.22 ).

Clinical Implications: Erectile function instruments must include items about erectile aids. Men who report that they have not attempted intercourse should not be assumed to have erectile dysfunction, but should be asked the reason why: for men who report lack of opportunity or preference for intercourse, the score of the three non-intercourse IIEF6 questions should be doubled.

Strengths \& Limitations: This is a large study of patients in a real-world setting. Although the study only includes radical prostatectomy patients, and although the study cohort is not fully representative of the US prostate cancer population as whole, these issues would not affect the key findings.

Conclusion: The IIEF6 can be redesigned to better assess the individual patient.

\section{Keywords}

Erectile function; IIEF; Patient reported outcomes; Prostate cancer; Radical prostatectomy; Sexual function

\section{Introduction}

The International Index of Erectile Function (IIEF) is the predominant patient-reported outcomes instrument for assessing male sexual function. A PubMed search in late 2018 for the IIEF retrieved over 3,500 hits overall, with over 800 clinical trials. The IIEF was used as the primary endpoint in all the initial trials of sildenafil, as well as for other therapies for erectile dysfunction, including PDE-5 inhibitors such as tadalafil and vardenafil, oral phentolamine, and intracavernosal injections[1].

There are five domains in the IIEF: erectile function, orgasmic function, sexual desire, intercourse satisfaction and overall satisfaction. The six items in the erectile function domain are routinely used as a stand-alone instrument (IIEF6)[1]. The Sexual Health Inventory for Men (SHIM, IIEF5), which is extensively used as a diagnostic tool for erectile dysfunction, uses 4 of the 6 questions on the IIEF6[2] and one question asking about sexual satisfaction. The IIEF6 and SHIM are of proven value for use at the population level. The psychometric properties have been well described[1] and accordingly, we should be confident that, for instance, a comparison of group means in a randomized trial will give reliable data on the relative value of two treatments for erectile dysfunction.

There are, however, three obvious and immediate problems with using the IIEF6 (or SHIM) in the clinic to evaluate an individual patient. First, there is no account of the use of "nonsystemic" erectile aids such as intracavernosal injections or a vacuum device. It is unclear whether a man who uses an erectile aid should complete the IIEF6 describing his experiences when using the aid or when not using the aid. Second, the IIEF6 includes three items about intercourse, with the response of "did not attempt intercourse" being given a 
score of 0 . Accordingly, a man not engaging in sexual intercourse in the previous four weeks - the recall period used in the IIEF - can obtain a maximum score of 15 on the 1 - 30 scale and will thus automatically be classified as having at least moderate erectile dysfunction. There are, of course, many reasons other than erectile dysfunction why a man may not engage in intercourse, such as lack of a partner[3] or preference for other types of sex in men who have sex with men[4]. Third, a man reporting no sexual activity in the past four weeks cannot have a score greater than 5 , in the "severe erectile dysfunction" range. It seems reasonable to suppose that some men with low libido have reasonable erectile function even if they do not engage in any sexual activity in a given four-week period. Moreover, even though the IIEF explicitly describes sexual activity as "intercourse, caressing, foreplay and masturbation", patients may vary in their understanding of those terms or, of course, not read the instructions carefully and equate sexual activity exclusively with intercourse[3].

At Memorial Sloan Kettering Cancer Center, (MSKCC), the IIEF6 is used as part of routine care in the evaluation and follow-up of men with prostate cancer. This provides a large database of patient responses to analyze the properties of the IIEF6 with respect to the three problems identified above: use of erectile aids, not having intercourse, and no sexual activity in a four-week period. The MSKCC patient-reported outcomes team made modifications to the IIEF6 in light of analyses of this database. Here we report the data that prompted us to modify the IIEF6 as well as the results of the IIEF after the modifications were implemented. Data are also analyzed from a systematic comparison of the IIEF6 and Expanded Prostate Cancer Index Composite (EPIC-26) sexual domain, undertaken to provide methods to interconvert scores between the two instruments[5].

\section{Methods}

The IIEF6 was implemented as part of routine clinical care for prostate cancer patients in the Department of Surgery on April of 2009. The current study includes all IIEF6 questionnaires completed by patients until the study database was closed on July 30, 2018. Radical prostatectomy patients complete the IIEF6, as well as questions about urinary function and quality of life[6], electronically, either by responding to an email at home or by using a tablet computer in the clinic. MSKCC made two substantive modifications to the instrument since it was first implemented. First, a question on use of injections was added. Patients are asked if they use injection therapy and if so, are administered one item from the IIEF6 concerning whether erections are sufficient for penetration. Patients are then instructed to complete the IIEF6 to describe their experience when not using injections, but with using oral medication such as Viagra, if they use it. Note that the IIEF6 was originally designed to evaluate erectile function with the use of medication, so details of function without use of medication is not asked. This approach was later modified to ask about erectile aids in general, rather than focusing only on injections. Second, a responsive item was added, administered only if patients stated that they had not attempted intercourse. This asked for the reason why the respondent had not attempted intercourse, with the options of lack of confidence or ability, lack of a willing partner or "other", with a free text box. Timing of the modifications and number of patients filling out each version of the questionnaire is given in Table 1, along with the time points. The exact wording of the new items is given in the supplementary appendix. Note that the version of the IIEF6 used at MSKCC involves some 
modifications of language as suggested by Kilbridge et al.[7] in order to make it appropriate for men across the socioeconomic spectrum.

The proportion of patients reporting use of erectile aids and their responses to the question about function when using the aid was calculated. We also sought to determine whether, before introduction of the erectile aids question, patients using such aids were giving responses as to erectile function either with or without the use of aids. It is possible to calculate the expected erectile function domain scores for each scenario by defining $\pi$ as the proportion of men using erectile aids, $x$ as the erectile function domain score in men who do not use erectile aides, $y$ as the erectile function domain score in men who use erectile aids answering the questions based on using erectile aids and $z$ as the erectile function domain score in men who use erectile aids answering the questions based on when they are not using erectile aids. The values $\pi, x, y$ and $z$ are estimated from the responses after implementation of the item on use of aids, with $y$ calculated as the response to the single item multiplied by 6 , so as to be on the same scale as the IIEF6. If, before the erectile aid question was implemented, respondents using aids gave function without use of the aids, expected score would be $\pi \times Z+(1-\pi) \times x$; expected score if respondents gave function with the use of aids would be $\pi \times y+(1-\pi) \times x$. A one-sample t-test was then used to determine whether observed erectile function domain scores were different to those expected under the two scenarios.

To develop a conversion factor to allow estimation of erectile function in a man who had not attempted sexual intercourse in the prior four weeks, but who reported that the reason was for a reason other than erectile dysfunction, the data obtained before and after implementation of the item on the reason for not attempting intercourse was analyzed. In men who reported attempting intercourse, the observed erectile function domain score was compared with a predicted erectile function domain score obtained by doubling the score of the three questions that did not ask about intercourse (IIEF Q15, Q1, and Q2, see appendix). The simple rationale for this approach is that half of the questions on the IIEF6 ask about intercourse and half do not. Observed and predicted scores were then compared by BlandAltman[8]. In the group of men who gave a response to the item concerning the reason for not attempting intercourse, scores on the IIEF6 non-intercourse questions were compared by reason.

For men reporting no sexual activity on to IIEF question 15, responses to the item "how do you rate your confidence that you could get and keep an erection?" were tabluated. To estimate erectile function in men who reported no sexual activity on IIEF6, data was used from a study to compare IIEF6 and MSKCC urinary function questionnaire with the EPIC-26. The study has been described in full[5]. In brief, men completing as part of routine care the IIEF6 (at MSKCC) or EPIC-26 (as part of the Michigan Urological Surgery Improvement Collaborative (MUSIC)) were asked if they would complete a second questionnaire for research purposes. If so, the two questionnaires were presented in random order.

As each survey response would not be considered independent, since surveys completed at different followup times by the same patient would be correlated, all confidence intervals 
were calculated using bootstrap methods. All analyses were performed using Stata 15 (StataCorp, College Station, TX). Ethical approval was obtained by a waiver for use of deidentified data collected for routine care from the MSKCC IRB.

\section{Results}

A total of 17,656 surveys were taken by 5,584 different men after questions on erectile aids were added to the IIEF6. Excluded were 190 surveys where patients did not answer regarding erectile aid usage and 848 where patients reported no sexual activity. Of these 16,618 surveys, erectile aid use was reported in $15 \%$ (95\% CI $\pm 0.5 \%$ ). Table 2 shows a breakdown of the aids used and reported function with and without the use of aids. Intracavernosal injections were by far the most common erectile aid used. Erectile aids resulted in men having an erection sufficient for penetration most or all of the time on $60 \%$ (95\% C.I. $58 \%, 62 \%)$ of surveys.

Of the 7,076 surveys completed before inclusion of the question on erectile aids, 5,450 were from men engaging in sexual activity, with a mean score was 17.7. Predicted score was 18.3 if patients using erectile aids reported their function when using aids and 16.7 if such patients reported function when not using aids. There was evidence of a difference between the two predicted scores and evidence that the observed mean score differed from both predicted scores (all p-values $<0.0001$ ). In the absence of clear instructions to the contrary, therefore, some men using erectile aids complete the IIEF6 describing their experience with the aids.

There were 11,874 surveys in which patients completed all six IIEF6 questions and reported attempting sexual intercourse. The Bland-Altman plot comparing the full erectile function domain score to a score derived from doubling the score for questions that did not ask about intercourse is given in the appendix. The correlation between the two scores was 0.98 . The mean difference was 0.059 (95\% CI 0.031, 0.088) higher in the score doubling the nonintercourse questions. Although statistically significant, the difference is tiny, constituting about $1 / 500^{\text {th }}$ of the scale. The limits of agreement was -3.10 to 3.22 , that is, the two IIEF scores are within 3 points for $95 \%$ of surveys.

Of the 3,006 complete surveys after the implementation of the question about reasons for not attempting in intercourse, 1,052 (35\%; 95\% CI $\sim \pm 2 \%$ ) reported no attempt at intercourse. Reasons for not attempting intercourse were given as lack of ability or confidence in 53\%, lack of willing and available partner in $28 \%$, "other" in $17 \%$, with $1 \%$ not answered. Free text responses are given in the appendix but include the respondent's health issues, partner's health issues, low libido, and preference for non-penetrative sex or for sex with men. Figure 1 shows the distribution of scores when doubling the three non-intercourse IIEF questions depending on the response to the intercourse question. Scores are always low for men who cite lack of ability or confidence as the reason for not attempting intercourse; scores have a uniform distribution, including some high scores, where the reason for not attempting intercourse is given as "lack of willing partner" or "other". 
Erectile function domain scores were calculated by imputing the scores for intercourse questions as 0 for men not attempting intercourse due to poor erectile function and as the mean of the first three questions for men not attempting intercourse reasons other than poor function. Using this approach, $10.2 \%$ (95\% CI $8.4 \%, 12.1 \%)$ of surveys where men reported that they did not attempt intercourse would be considered as having good erectile function using the cutpoint of 24[9].

To illustrate the effects of taking into account reason for not having intercourse, figure 2 shows erectile function domain scores over time for six men recovering erectile function after prostate cancer surgery and who reported no attempts at intercourse due to lack of willing partner at one or more-time points. It can be seen that the use of the adjusted scores gives results that appear more in keeping with a patient's likely course of recovery.

To examine function in men who claimed no sexual activity, data was analyzed from 1224 surveys in which patients responded to the confidence question and the sexual activity question on the IIEF6 and also completed the EPIC-26. Patients reported that they did not engage in any sexual activity on $20 \%$ of surveys (95\% CI $\sim 2 \%$ ). Figure 3 shows the distribution of EPIC sexual function score among these patients. Clearly, some men reported good sexual function on EPIC despite reporting no sexual activity on IIEF6. Among the 552 surveys where patients have a score on EPIC of at least 50, 507 (92\%) reported "fair" or better ability to have an erection, $531(96 \%)$ reported "fair" or better ability to have an orgasm, 494 (89\%) had erections firm enough for sexual activity, 461 (84\%) had erections more than half the time or always when they wanted one, $358(65 \%)$ reported good or very good ability to function sexually and $355(64 \%)$ reported that sexual function was only a very small problem or no problem at all.

Of the surveys in which no sexual activity was reported, $4.8 \%$ reported "good" or "very good" confidence to get an erection. Among 6,607 surveys where patients engaged in sexual activity and reported having high ("Good" or "Very Good") confidence that they could get and keep and erection, $84 \%$ (95\% CI $\pm 1 \%$ ) were considered to have good erectile function (adjusted erectile function domain score 224 ). The median (IQR) IIEF score for patients with good confidence was $25(23,28)$ whereas it was $30(29,30)$ for patients with very good confidence.

\section{Discussion}

We implemented and validated several changes to the IIEF6 in a large group of patients completing the instrument as part of routine care. Our findings might well be considered simple common sense: men who use erectile aids such as intracavernosal injections have different erectile function when they use the aid; erectile dysfunction is not the only reason why men do not attempt intercourse; some men do not engage in any sexual activity over a four-week period but do not have erectile dysfunction. That said, there have not been many previous attempts to modify erectile or sexual function instruments accordingly.

We make the following recommendations. First, any attempt to determine a man's erectile function must first ask whether he is using erectile aids and then obtain responses to 
questions about function when he is not using the aids. Second, men who report that they have not attempted intercourse in the prior four weeks should not be assumed to have erectile dysfunction, but should be asked the reason why. The erectile function domain score for men who state that they have not attempted intercourse for reasons other than erectile dysfunction (for instance, they do not have a partner, do not have penetrative sex or have sex with men) should be calculated by doubling the score of the first three questions on IIEF6, which do not reference intercourse. Similar scoring adjustments should be considered for other instruments that ask about intercourse, such as the long form version of the EPIC and the UCLA Prostate Cancer Index, should make similar scoring adjustments. Third, a man who reports "good" or "very good" confidence to have an erection, but who did not engage in sexual activity in the past four weeks, should be not automatically be considered to have erectile dysfunction.

Our findings also demonstrate why IIEF6 has good psychometric properties. In brief, such properties are measured as population averages and are not importantly affected by smaller subgroups. For instance, we estimate that of the $35 \%$ of men who report no intercourse - and who therefore cannot obtain a score on the IIEF6 sufficient to meet criteria for good erectile function - about $10 \%$ do not have erectile dysfunction. Our recommendation to include a question about the reason for lack of intercourse will therefore only affect $\sim 3.5 \%$ of men. Misestimation of erectile function in these men would not importantly affect psychometric statistics such as correlation with known predictors of erectile function. Our recommendations are important for the individual patient, but will not make much difference to group averages.

These small modifications to the IIEF are worth implementing to make it of value to a wider cross-section of our patients.

Two possible limitations of our study are that it included radical prostatectomy patients only and that the MSKCC population is not fully representative of the US population as a whole. That said, these issues would be unlikely to affect the key findings. For instance, the phenomenon of gay men who report no intercourse despite good erectile functioning is clearly not solely characteristic of radical prostatectomy patients; similarly, ethnic or educational status would not influence recommendations to ask men about use of erectile aids.

There are several further avenues of research that should be pursued. First, we recommend investigation of follow-up questions for men who report high or very high confidence in getting an erection but who report no sexual activity. Although it is likely that these men do not have erectile dysfunction, it is possible that additional items, such as presence of nocturnal erections, or sexual activity over a longer time-frame than the 4 weeks used in the IIEF, could refine estimates of erectile function in these men. We also believe it would be worth investigating the properties of the IIEF6 for men who have sex with men. The IIEF6 includes questions both about intercourse, which is viewed by many as referring exclusively to insertion of the penis into the vagina, and about penetration, which is not an important part of sexual activity for some men. 
In conclusion, several relatively minor changes to the IIEF can importantly improve its ability to estimate erectile function in individual patients.

\section{Supplementary Material}

Refer to Web version on PubMed Central for supplementary material.

\section{Acknowledgements:}

This work was supported in part by funds from the Sidney Kimmel Center for Prostate and Urologic Cancers, a Specialized Programs of Research Excellence grant (P50-CA92629) from the National Cancer Institute to Dr. Howard Scher and a National Institutes of Health/National Cancer Institute Cancer Center Support Grant (P30CA008748) to Memorial Sloan Kettering Cancer Center.

\section{References}

[1]. Rosen RC, Cappelleri JC, Gendrano N 3rd. The International Index of Erectile Function (IIEF): a state-of-the-science review. Int J Impot Res. 2002;14:226-44. [PubMed: 12152111]

[2]. Rosen RC, Cappelleri JC, Smith MD, Lipsky J, Pena BM. Development and evaluation of an abridged, 5-item version of the International Index of Erectile Function (IIEF-5) as a diagnostic tool for erectile dysfunction. Int J Impot Res. 1999;11:319-26. [PubMed: 10637462]

[3]. Rynja S, Bosch R, Kok E, Wouters G, de Kort L. IIEF-15: unsuitable for assessing erectile function of young men? The journal of sexual medicine. 2010;7:2825-30. [PubMed: 20487234]

[4]. Coyne K, Mandalia S, McCullough S, Catalan J, Noestlinger C, Colebunders R, et al. The International Index of Erectile Function: development of an adapted tool for use in HIV-positive men who have sex with men. The journal of sexual medicine. 2010;7:769-74. [PubMed: 19912494]

[5]. Singh K, Tin AL, Dunn RL, Kim T, Vickers AJ. Development and Validation of Crosswalks for Patient-reported Sexual and Urinary Outcomes Between Commonly Used Instruments. Eur Urol. 2018.

[6]. Vickers AJ, Savage CJ, Shouery M, Eastham JA, Scardino PT, Basch EM. Validation study of a web-based assessment of functional recovery after radical prostatectomy. Health Qual Life Outcomes. 2010;8:82. [PubMed: 20687938]

[7]. Kilbridge KL, Fraser G, Krahn M, Nelson EM, Conaway M, Bashore R, et al. Lack of comprehension of common prostate cancer terms in an underserved population. J Clin Oncol. 2009;27:2015-21. [PubMed: 19307512]

[8]. Bland JM, Altman DG. Agreement between methods of measurement with multiple observations per individual. J Biopharm Stat. 2007;17:571-82. [PubMed: 17613642]

[9]. Terrier JE, Mulhall JP, Nelson CJ. Exploring the Optimal Erectile Function Domain Score Cutoff That Defines Sexual Satisfaction After Radical Prostatectomy. J Sex Med. 2017;14:804-9. [PubMed: 28583341] 

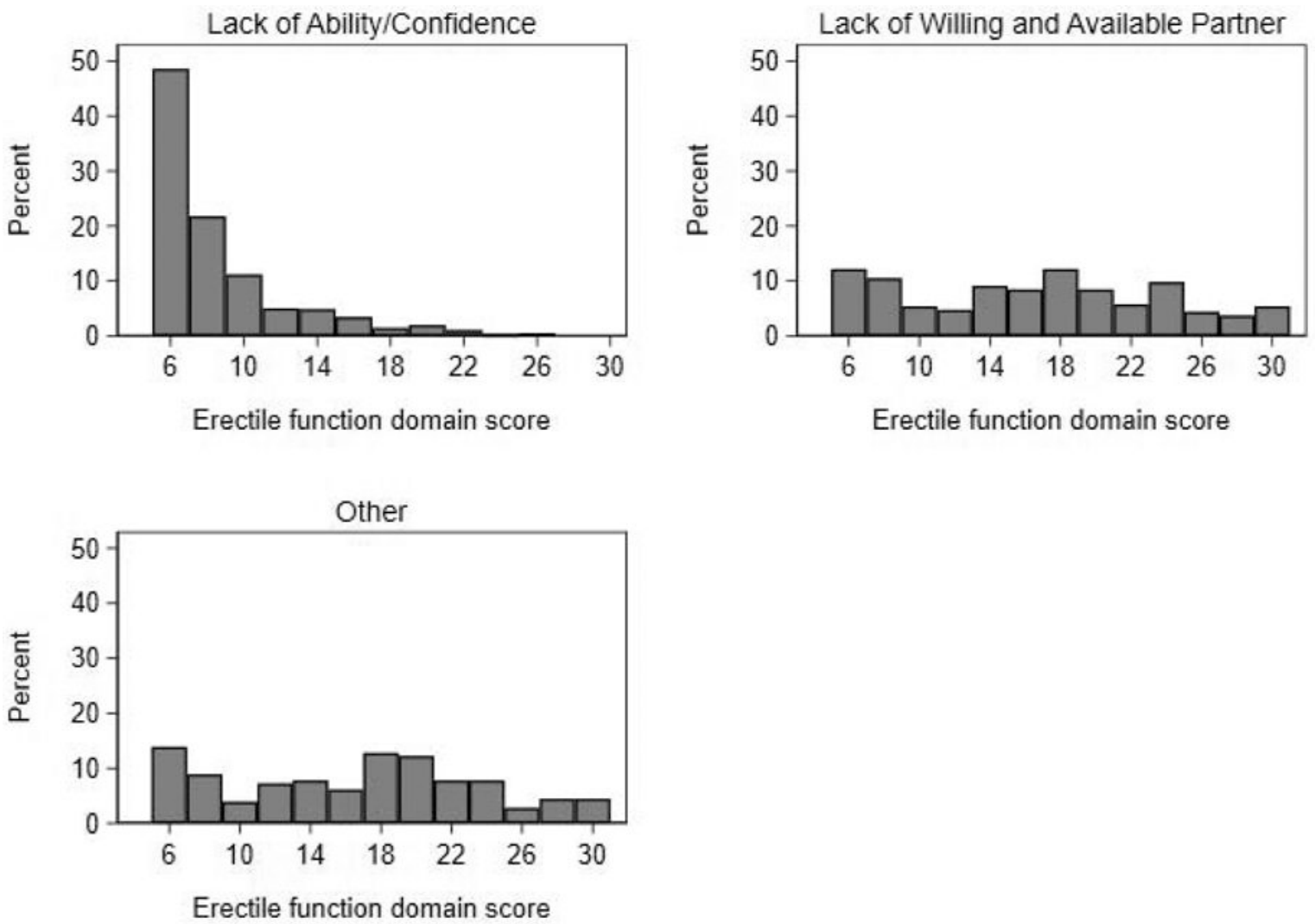

Figure 1.

Distribution of score for doubling the 3 non-intercourse questions based on response to reason for not attempting intercourse. 

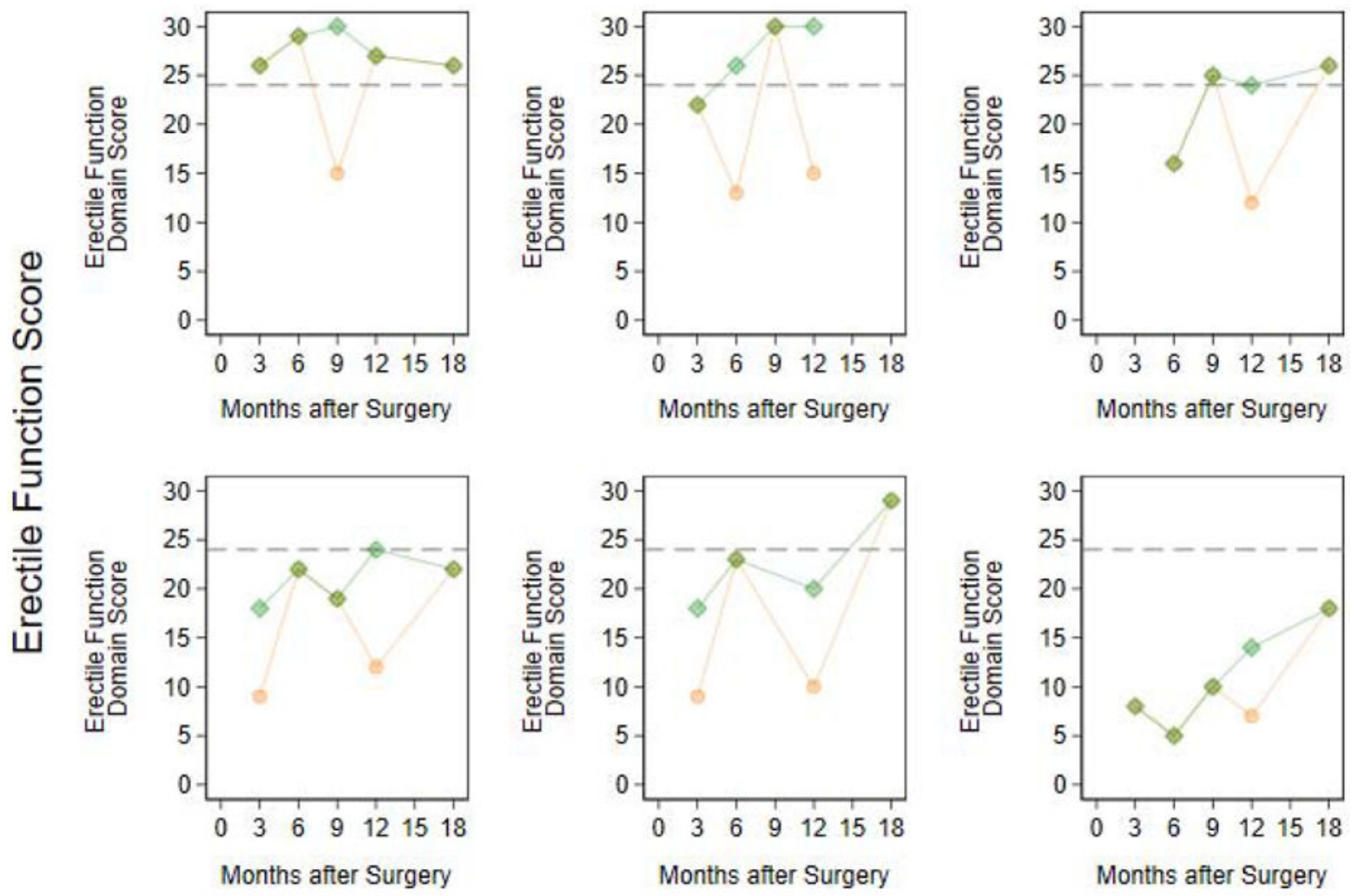

\section{Months after surgery}

Figure 2.

Erectile function domain scores over time for select six men recovering erectile function after prostate cancer surgery. Orange corresponds to standard erectile function domain score. Green corresponds to doubling the score of three non-intercourse questions when patients did not attempt intercourse due to lack of willing partner. Horizontal dashed-line indicates a standard cutoff for "good" erectile function. 


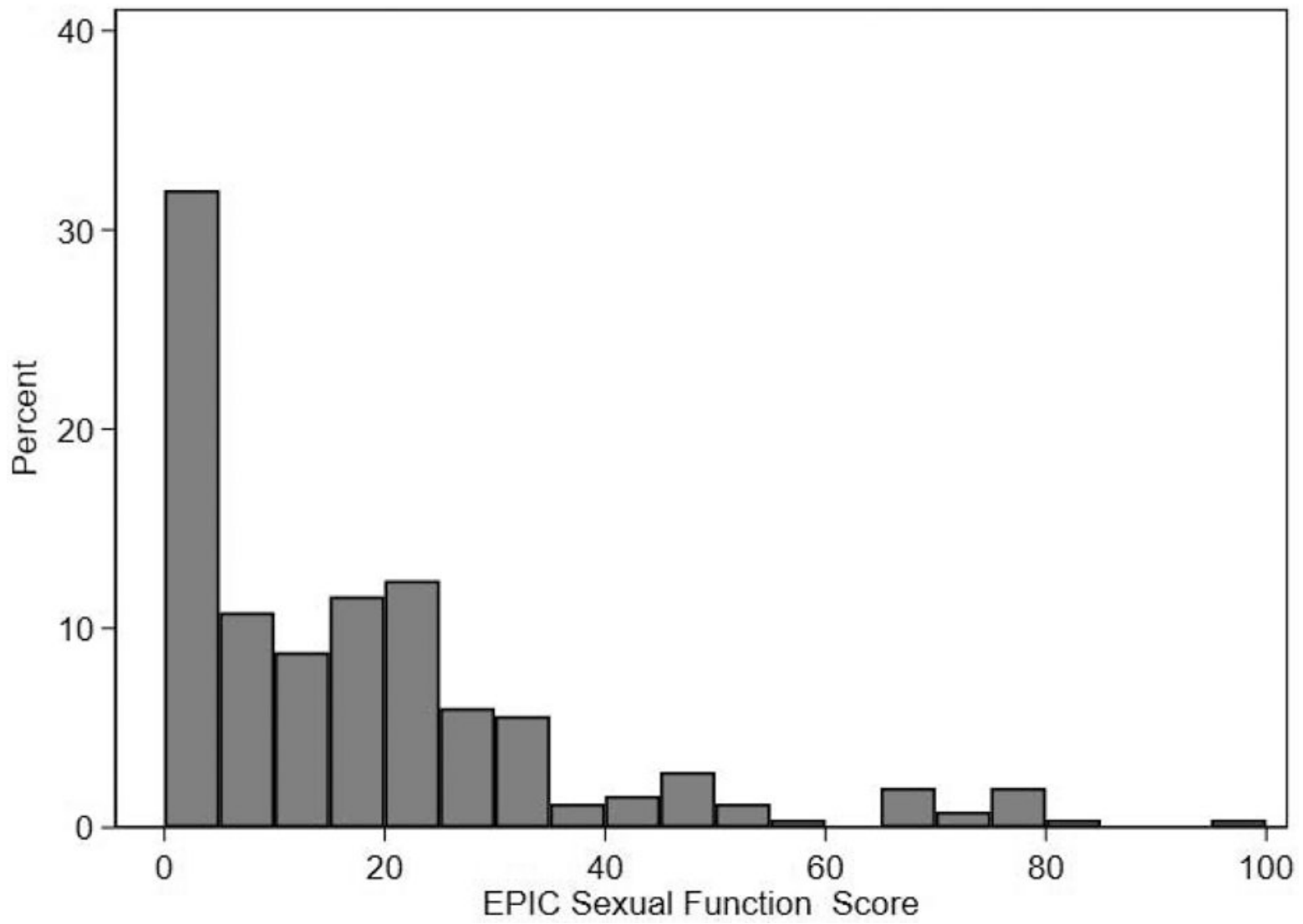

Figure 3.

Distribution of Expanded Prostate Cancer Index Composite (EPIC) sexual function score among patients reporting no sexual activity on the IIEF6 
Table 1.

Survey details based on modifications.

(Overall 24,732 surveys completed by 6,780 patients after radical prostatectomy).

\begin{tabular}{|c|c|c|c|}
\hline Survey Modification Type & $\begin{array}{l}\text { Prior to } \\
\text { Addition of } \\
\text { Injection } Q \\
(N=7,076 ; 29 \%)\end{array}$ & $\begin{array}{l}\text { Addition of } \\
\text { Injection } Q \\
(\mathbf{N}=13,524 ; 55 \%)\end{array}$ & $\begin{array}{l}\text { Updated } \\
\text { Injection } Q \\
\text { and Addition } \\
\text { of Partner } Q \\
(\mathrm{~N}=4,132 ; 17 \%)\end{array}$ \\
\hline Date of Earliest Survey & April 3, 2009 & March 18, 2012 & March 1, 2017 \\
\hline Number of Patients & 2,805 & 4,680 & 2,117 \\
\hline \multicolumn{4}{|l|}{ Survey Timepoint after surgery } \\
\hline 3-6 months & $1,901(27 \%)$ & $4,399(33 \%)$ & $1,262(31 \%)$ \\
\hline 9-15 months & $1,717(24 \%)$ & $3,348(25 \%)$ & $1,098(27 \%)$ \\
\hline 18-24 months & $1,726(24 \%)$ & $2,900(21 \%)$ & $1,040(25 \%)$ \\
\hline 3 years and later & $1,732(24 \%)$ & $2,877(21 \%)$ & $732(18 \%)$ \\
\hline \multicolumn{4}{|l|}{ Nerve-sparing Status } \\
\hline None & $351(13 \%)$ & $575(12 \%)$ & $236(11 \%)$ \\
\hline Unilateral & $439(16 \%)$ & $886(19 \%)$ & $476(22 \%)$ \\
\hline Bilateral & $1974(70 \%)$ & $3113(67 \%)$ & $1366(65 \%)$ \\
\hline Unknown & $41(1.5 \%)$ & $106(2.3 \%)$ & $39(1.8 \%)$ \\
\hline Adjuvant Hormonal Treatment ${ }^{a}$ & $80(2.9 \%)$ & $197(4.2 \%)$ & $94(4.4 \%)$ \\
\hline Unknown & $1(<0.1 \%)$ & $2(<0.1 \%)$ & $1(<0.1 \%)$ \\
\hline Adjuvant Radiation Treatment ${ }^{a}$ & $48(1.7 \%)$ & $94(2.0 \%)$ & $32(1.5 \%)$ \\
\hline Salvage Hormonal Treatment $b$ & 231 & 484 & 221 \\
\hline Unknown & 1 & 2 & 1 \\
\hline Salvage Radiation Treatment $b$ & 323 & 597 & 234 \\
\hline
\end{tabular}

${ }^{a}$ Adjuvant treatment defined as within 6-months of radical prostatectomy.

$b$ Percentages not shown for salvage treatment since patients have different amounts of followup. 
Table 2.

Breakdown of erectile aide usage and function with and without use of aids.

\begin{tabular}{|c|c|c|}
\hline & No use of erectile aid & Erectile aid use \\
\hline Number of Surveys & $14,122(85 \%)$ & $2,496(15 \%)$ \\
\hline Erectile aid & & $2,429(97 \%)$ \\
\hline Intracavernosal injections & & \\
\hline Vacuum Device $^{a}$ & & $51(8.0 \%)$ \\
\hline Urethral Suppository $^{a}$ & & $4(0.6 \%)$ \\
\hline Other $^{a}$ & & $25(3.9 \%)$ \\
\hline More than 1 aid ${ }^{a}$ & & $13(2.1 \%)$ \\
\hline Erectile function domain scores (without aid) $(\mathrm{N}=13470)$ & $17(7,26)$ & $8(6,18)$ \\
\hline \multicolumn{3}{|l|}{ "Erection sufficient for penetration" with use of aid } \\
\hline No sexual activity & & $259(10 \%)$ \\
\hline Almost never & & $306(12 \%)$ \\
\hline Less than half the time & & $203(8.1 \%)$ \\
\hline About half the time & & $221(8.9 \%)$ \\
\hline More than half the time & & $456(18 \%)$ \\
\hline Almost always & & $1,034(41 \%)$ \\
\hline Unknown & & $17(0.7 \%)$ \\
\hline
\end{tabular}

${ }^{a}$ Detailed erectile aids beyond intracavernosal injections were only captured in patients who answered a revised version of the survey (n=634) 